\title{
The Ion Mass Imager on the Planet-B spacecraft
}

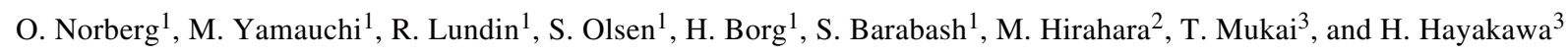 \\ ${ }^{1}$ Swedish Institute of Space Physics, Box 812, SE-981 28 Kiruna, Sweden \\ ${ }^{2}$ Rikkyo University, Tokyo, Japan \\ ${ }^{3}$ The Institute of Space and Astronautical Science (ISAS), Japan
}

(Received July 28, 1997; Revised December 2, 1997; Accepted January 5, 1998)

\begin{abstract}
The Ion Mass Imager (IMI) is a light-weight ion mass composition instrument for the Japanese Planet-B mission to be launched to Mars in 1998. The objective of the Planet-B mission is to study the Martian environment with emphasis on the upper atmosphere interaction with the solar wind. IMI measures positive ions with energies between $10 \mathrm{eV} / \mathrm{q}$ and $35 \mathrm{keV} / \mathrm{q}$ and with a mass resolution high enough to resolve the most important ion species $\left(\mathrm{H}^{+}, \mathrm{He}^{++}, \mathrm{He}^{+}, \mathrm{O}^{++}, \mathrm{O}^{+}, \mathrm{O}_{2}^{+}\right)$. The instrument has a $360^{\circ}$ field-of-view aperture and uses the spacecraft spin to cover almost the full unit sphere to obtain three-dimensional distribution functions every $4 \mathrm{~s}$ (half a spacecraft spin period). Particles are energy-filtered by a spherical electrostatic analyzer, and then mass-analysed by the magnetic separation method. The ions hit a microchannel plate assembly with a position sensitive anode divided into 32 mass channels. Together with 16 angular sectors, this system "images" the direction of motion and mass of ions. A preacceleration voltage of $0-4000 \mathrm{~V}$ is used to select the mass range, e.g., modes optimized for light ions (up to $\mathrm{O}^{+}$) and heavy ions $\left(\mathrm{O}^{+}\right.$to charged dust grains). A loss-less data compression algorithm is used in the in-flight processing software to optimize the amount of data that can be returned from Mars.
\end{abstract}

\section{Introduction}

The Martian plasma environment has been explored by the Mars 2, 3, 4, 5, and Phobos-2 spacecraft. Many important plasma boundaries were discovered such as a bow-shock, magnetopause, magnetospheric cavity, and a plasma tail. A boundary layer with a low-energy component of presumably ionospheric origin was also observed near Mars. Yet, the Martian plasma environment still remains somewhat of a puzzle. The Martian magnetosphere, as defined by the region of reduced plasma flow, is more extended than previously anticipated. It is dominated by ions of Martian origin, e.g., $\mathrm{O}^{+}$and molecular ions, and has several characteristics similar to that of the solar wind interactions with comets (Lundin et al., 1990). Figure 1 shows a simplified model of the Martian "magnetosphere", based on previous in-situ plasma measurements. One objective of the Japanese Planet-B Mars mission is to clarify these matters.

Because of the very low intrinsic magnetic field of Mars, the Martian upper atmosphere (dominated by $\mathrm{O}_{2}^{+}$) is exposed to the solar wind. This implies that ionization in the upper atmosphere is due to both solar UV and solar wind plasma interaction. The latter may take several forms, e.g., a viscous-like interaction, and the critical velocity ionization by the solar wind interaction with the neutral atmosphere. Meanwhile, Phobos- 2 found an escape of plasma from Mars (outflow of atmospheric oxygen), of the order $1 \mathrm{~kg} / \mathrm{s}$ (Lundin and Dubinin, 1992).

Another interesting topic to study is the solar wind interaction with the two Martian satellites, Phobos and Deimos.

Copy right $(\subset$ The Society of Geomagnetism and Earth, Planetary and Space Sciences (SGEPSS); The Seismological Society of Japan; The Volcanological Society of Japan; The Geodetic Society of Japan; The Japanese Society for Planetary Sciences.
The moons have a low mass density $\left(1.8 \pm 0.5 \mathrm{~g} / \mathrm{cm}^{3}\right.$ for Phobos), indicating that they contain a significant fraction of volatile elements (e.g., $\mathrm{H}_{2} \mathrm{O}$ and $\mathrm{CO}_{2}$ ice). If so, a very thin atmosphere is expected to form, most likely on the sunfacing side and in particular after meteorite impacts. This explains the observation from the Phobos-2 spacecraft of the cometary behaviour of Phobos and Deimos as well as the indications of an oxygen corona in the Phobos orbit. Ionization of these molecules due to interaction with the solar wind definitely plays an important role for the escape of volatile elements.

One fascinating property of the planetary environments is the existence of ring structures. Although these are known to exist around the giant planets, there are speculations about ring structures also around Mars (Dubinin et al., 1990). Micro-meteorite impacts on the Phobos surface may substantially contribute to the hot oxygen corona around Mars. Study of the "dusty" plasma behaviour is interesting from a cosmogonic point of view too. Fine grains with mass up to $10^{12}$ a.m.u. may, if electrically charged, be strongly controlled by magnetic and electric forces. Such a "dusty" plasma is then likely to be the source of larger grains which eventually accrete into larger bodies. Magnetic and electric forces are therefore expected to control matter over an extended period of time throughout the formation of a planetary system. Therefore, measuring dusty plasma is also an important scientific objective.

\section{Scientific Objectives}

Primary and secondary scientific objectives of the studies of the Martian plasma environment are summarised in Table 1 together with the related previous works. IMI will be operated together with the other scientific instruments on 


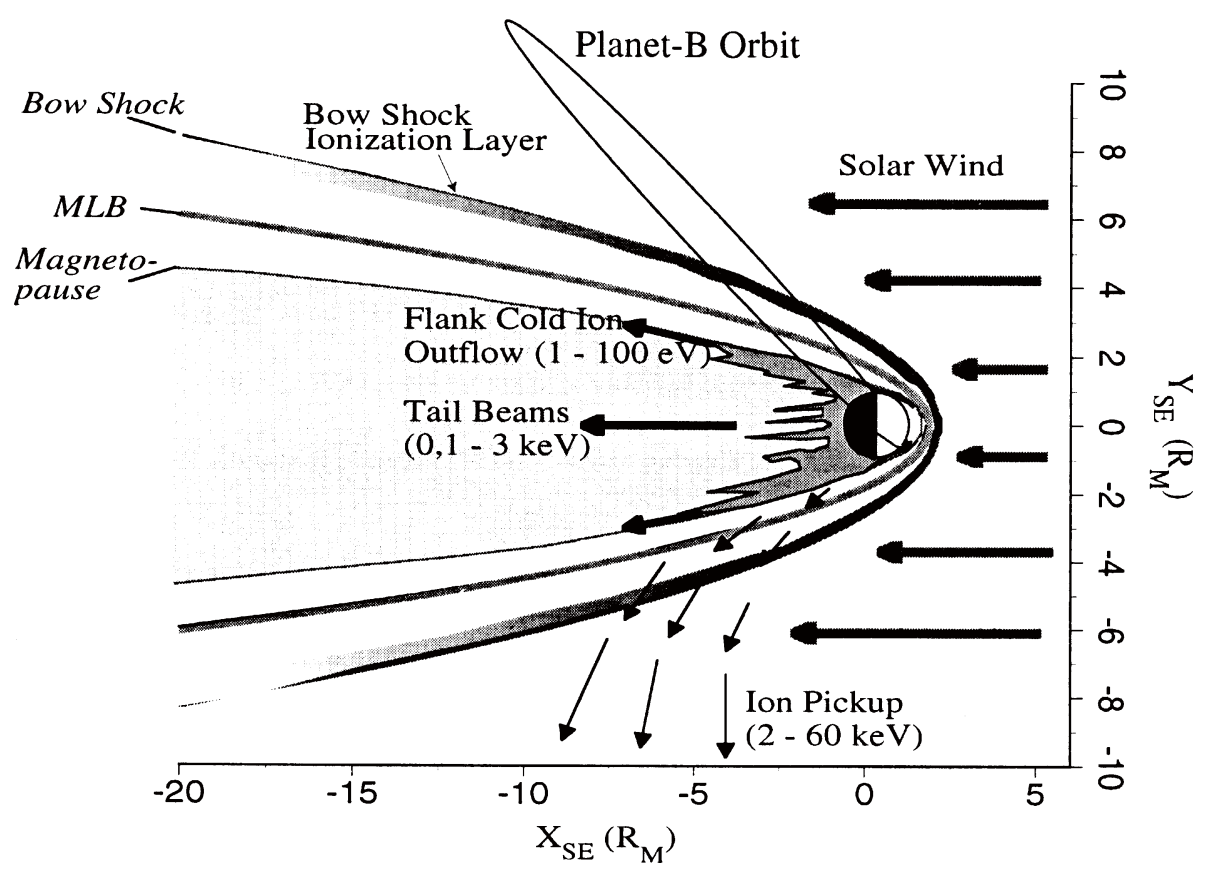

Fig. 1. A model of the Martian "magnetosphere" based on the measurements made using the ASPERA experiment on-board Phobos-2. The massloading boundary (MLB) was one of the features discovered during the Phobos-2 mission (Lundin et al., 1990). A representative orbit of the Planet$\mathrm{B}$ spacecraft is indicated in the figure.

Table 1(a). Primary scientific objectives of the IMI instrument on the Planet-B spacecraft.

\begin{tabular}{|c|c|c|c|}
\hline Primary objectives & Past works & Primary instruments & $\begin{array}{l}\text { Supplemental } \\
\text { instruments }\end{array}$ \\
\hline Non-thermal ion escape & $\begin{array}{l}\text { Lundin et al., 1989a; } \\
\text { Barabash et al., } 1995\end{array}$ & all plasma & B, wave, dust \\
\hline Tail ion outflow & $\begin{array}{l}\text { Gringauz et al., 1976; } \\
\text { Vaisberg, } 1976\end{array}$ & all plasma & B, wave, dust \\
\hline Acceleration processes & $\begin{array}{l}\text { Barabash et al., 1991; } \\
\text { Dubinin et al., 1993; } \\
\text { Lundin et al., } 1993\end{array}$ & all plasma & B, wave, dust \\
\hline Filamental structures & Lundin et al., 1993 & all plasma & $\mathrm{B}$, wave, dust \\
\hline $\begin{array}{l}\text { Boundaries } \\
\text { (bow-shock, ionopause, mass-loading boundary) }\end{array}$ & $\begin{array}{l}\text { Gringauz et al., 1976; } \\
\text { Vaisberg and Smirnov, 1986; } \\
\text { Lundin et al., 1993; } \\
\text { Dubinin et al., } 1996\end{array}$ & all plasma, B & wave \\
\hline $\begin{array}{l}\text { Ionosphere } \\
\text { (SW interaction, dawn-dusk asymmetry) }\end{array}$ & $\begin{array}{l}\text { Intrilligator and Smith, 1979; } \\
\text { Ip et al., } 1993\end{array}$ & all plasma & B, wave, Camera \\
\hline Phobos and Deimos (dust rings) & $\begin{array}{l}\text { Ip, 1988; Dubinin et al., 1990; } \\
\text { Barabash and Lundin, } 1994\end{array}$ & dust counter, all plasma & B, waves, Camera \\
\hline Intrinsic B-field & $\begin{array}{l}\text { Riedler et al., 1989; } \\
\text { Dolginov et al., 1976; } \\
\text { Yeroshenko et al., } 1990\end{array}$ & $\mathrm{~B}$ & all plasma \\
\hline
\end{tabular}

Planet-B to fulfil the overall scientific objective of the mission, to study the Martian environment with emphasis on the upper atmosphere interaction with the solar wind. Specifically, IMI will be used to address the question of ion composition in the Martian environment, non-thermal ion escape, and ion pick-up by the solar wind. The objective to study possible dust rings around Mars will also be addressed by IMI, since the instrument has a wide mass range covering a dusty plasma component. Identification of the flow direction is very important, necessitating an instrument with three-dimensional (3D) coverage. 
Table 1(b). Secondary objectives of the IMI instrument on the Planet-B spacecraft.

\begin{tabular}{|c|c|c|c|}
\hline Secondary objectives & Past works & Primary instruments & $\begin{array}{l}\text { Supplemental } \\
\text { instruments }\end{array}$ \\
\hline Solar wind dynamics & Barabash et al., 1993 & & \\
\hline perpendicular heating + cavitons in weak B-fields & (comparison with the Earth) & all plasma, wave & $\mathrm{B}$ \\
\hline Acceleration by parallel $E$ in weak B-fields & (comparison with the Earth) & all plasma, wave & $\mathrm{B}$ \\
\hline bi-directional electron/ion fluxes in weak B-fields & (comparison with the Earth and the heliosphere) & all plasma, B & wave \\
\hline Ion outflow at the poles & Lundin et al ., 1989a & all plasma & B, wave \\
\hline
\end{tabular}

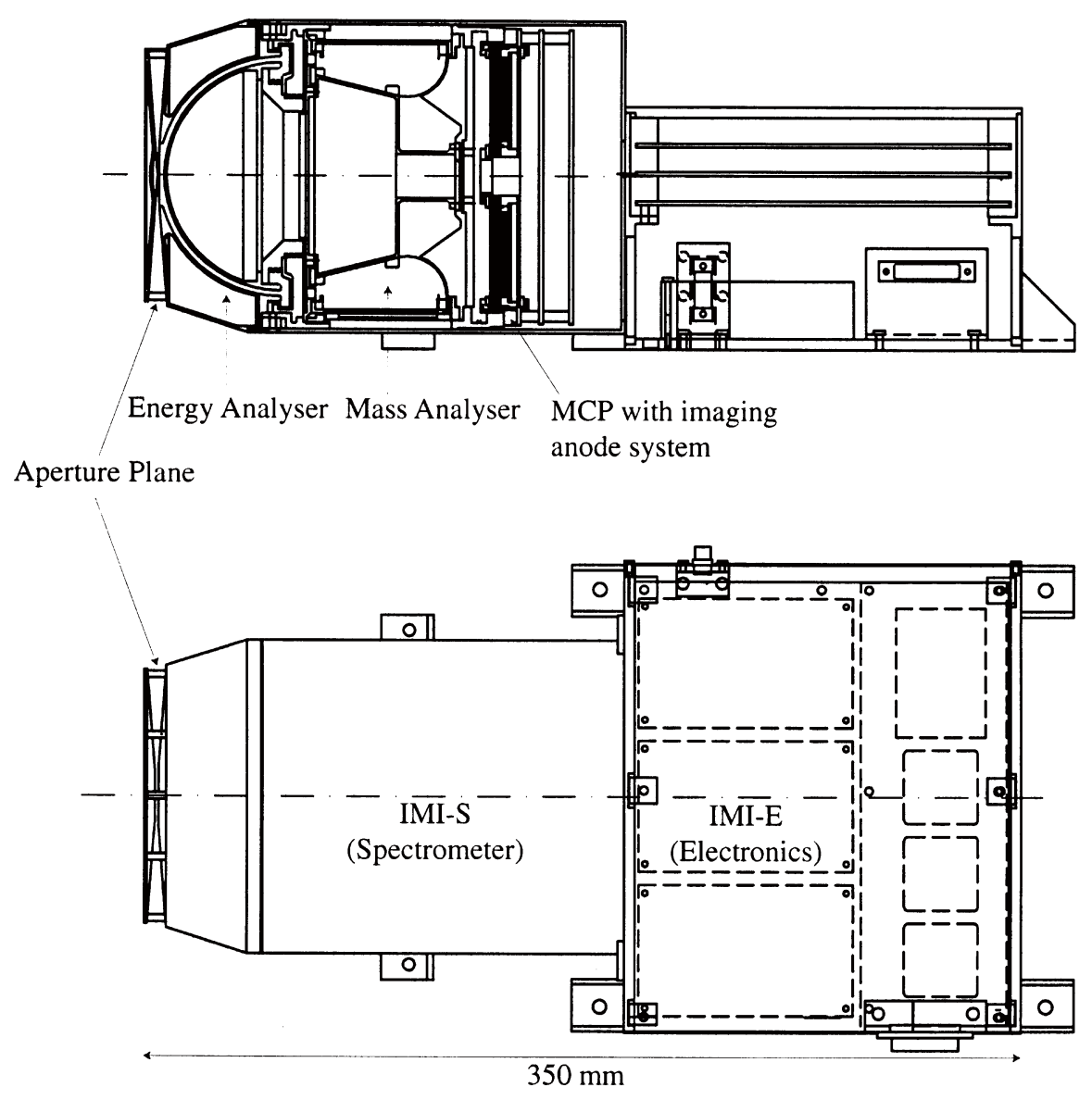

Fig. 2. Two views of IMI showing the internal design of the spectrometer.

\section{Instrument Description}

The IMI instrument has a heritage from the design, manufacture, test, and flight of two earlier versions of this type of instrument. Those are the TICS instrument flown on the Swedish-German magnetospheric-ionospheric research satellite Freja which was operated 1992-1996 (Eliasson et al., 1994), and the IMIS instrument which was developed for the ill-fated Mars-96 spacecraft. The instrument also has a heritage from the ASPERA experiment flown on the Soviet spacecraft Phobos-2 to Mars (Lundin et al., 1989b).

IMI consists of two parts (see Fig. 2), the cylindrical sensor unit (IMI-S) which contains an energy analyzer and a mass spectrometer, and the electronics unit (IMI-E) containing all processing, memory, power conditioning, telemetry, and command functions. The IMI functional block diagram is shown in Fig. 3. IMI is located in one of the corners of the Planet-B spacecraft body, with the normal vector of the aperture plane perpendicular to the spacecraft spin axis. This location ensures that IMI will cover almost the whole $4 \pi$ unit sphere in half a spacecraft spin, or in roughly $4 \mathrm{~s}$. A small part of the field-of-view will be shadowed by one of Planet-B's solar arrays.

\subsection{The sensor unit (IMI-S)}

IMI covers the energy range $10 \mathrm{eV} / \mathrm{q}$ to $35 \mathrm{keV} / \mathrm{q}$. It consists of a spherical electrostatic energy analyzer, a mass analyzer featuring a cylindrical magnetic field, and a microchannel plate (MCP) assembly with an imaging (position sensitive) anode. Particles enter the energy filter, a spherical "top hat" electrostatic analyzer, through a $360^{\circ}$ by $5^{\circ}$ aperture plane. The dimensions of the electrostatic analyzer are: $43.9 \mathrm{~mm}$ (inner radius), $46.1 \mathrm{~mm}$ (outer radius), and $48.3 \mathrm{~mm}$ (top hat radius). The energy analyzer allows the passage of ions with energies within a prescribed passband. The outer hemisphere of the energy analyzer is kept at 


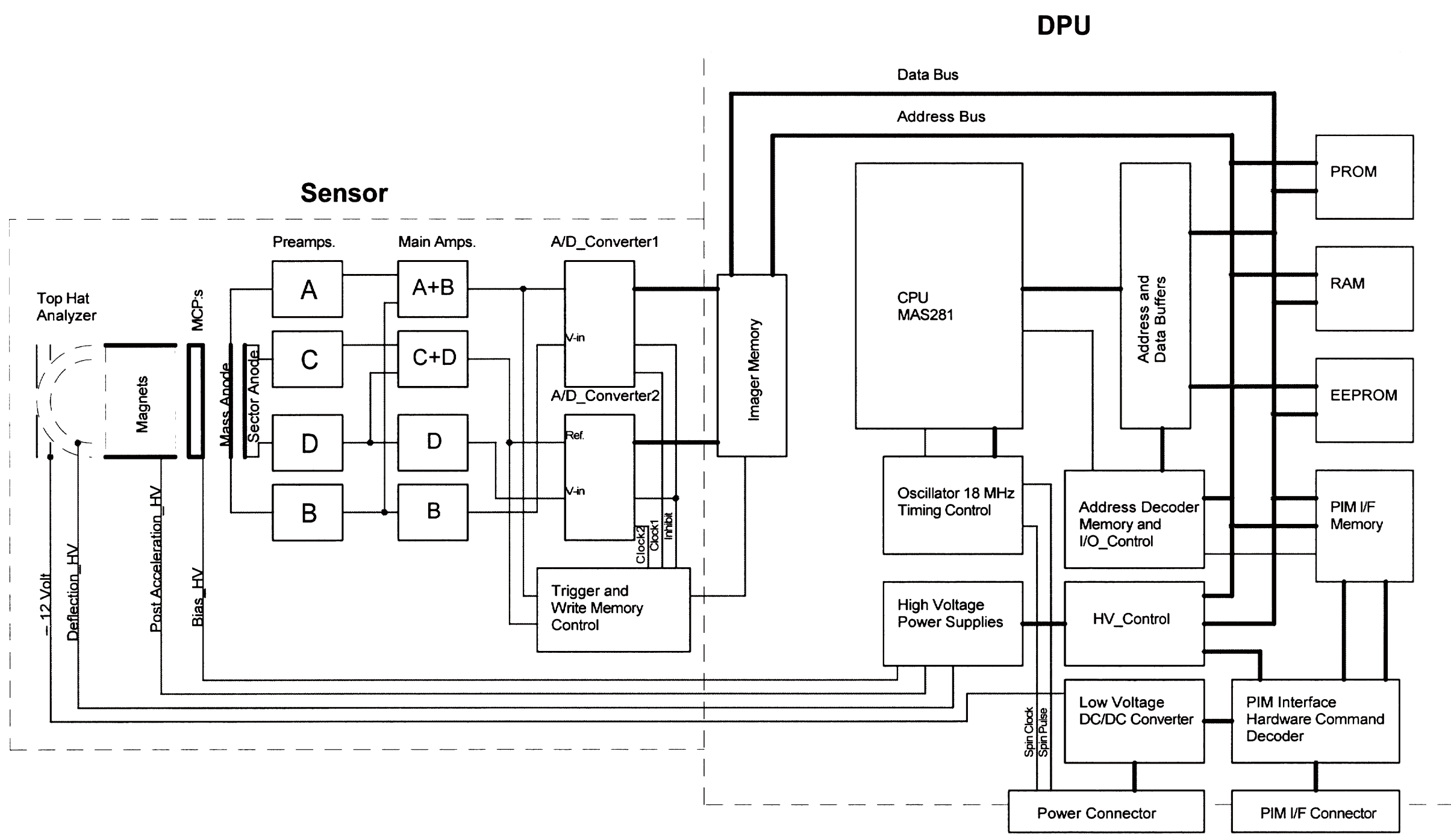

Fig. 3. Functional block diagram of the IMI instrument. The "top-hat" surface facing towards space is at ground (spacecraft) potential. 
a potential of $-12 \mathrm{~V}$, whereas the potential of the inner hemisphere (deflection potential) is stepped from $-12 \mathrm{~V}$ to $-4 \mathrm{kV}$ through 32 exponentially spaced settings with minimum step of $1 \mathrm{~V}$.

Ions transmitted through the energy analyzer enter the mass analyzer through a $1 \mathrm{~mm}$ wide slit. The mass analyzer uses a cylindrical magnetic field which deflects the ions inward, toward the central axis of the analyzer system. The magnetic system consists of 16 radially oriented permanently magnetised Neodymium-Iron-Boron magnets. All magnets are equivalent within $\pm 1 \%$ accuracy. The magnetic field strength in the mass analyzer is $\approx 0.11 \mathrm{~T}$. The dimensions of the magnet assembly and the field strength has been optimized so that all ions with energies greater than $\approx 2.3 \mathrm{keV} / \mathrm{q}$ will pass through the analyzer and produce a "mass image" on the MCP. The whole magnet assembly can be biased with a pre-acceleration voltage between 0 and $4 \mathrm{kV}$, of which a 2.3 $\mathrm{kV}$ setting will ensure all ions, independent of mass, reach the MCP. The use of a magnetic analyzer and an adjustable pre-acceleration makes the instrument simple and dependable. The pre-acceleration can be used to vary the mass resolution of the instrument.

Particles striking the front of the $100 \mathrm{~mm}$ diameter MCP (two stacked plates biased at $-2.3 \mathrm{kV}$ on the front side) produce an electron shower at the back side. The electrons are detected by an "imaging" anode system. A system of 32 concentrical rings behind the MCP measures the radial impact position (representing ion mass), whereas 16 sector anodes measure the azimuthal impact position (representing ion entrance angle). The ring and sector systems are each interconnected by a resistor chain. The charge pulse produced by the electron shower expands from the impact position in both directions of the chain. Attenuated by the resistor chain, the pulses reaching the ends of the chain will have different amplitude depending on how many resistors they have passed through. At the end of the ring and sector chains charge sensitive Amptek A250 pre-amplifiers measure the end point charge pulses. The ratio between the pulse produced in one end of a chain and the sum of the pulses in both ends is fed into fast analog-to-digital (A/D) converters. The A/D converter outputs are linear functions of the impact position on the ring and sector anodes. The A/D converter outputs from both chains are then used to point a register address representing the ring-sector combination which is incremented beforehand. The registers are allowed to sample pulses during $13.6 \mathrm{~ms}$ interval after which they are read out and reset.

A new feature of the IMI instrument is the temperatureadjusted voltage algorithm. Since we have only one highvoltage supply for the MCP bias, pre-acceleration, and deflection system, we use the opt-coupler and also insert an extra register to each system. This causes fluctuation of applying voltage over the system because the impedance of the MCP depends on the temperature, and the performance

Table 2. The six operational mode groups.

\begin{tabular}{lll}
\hline Mode & Description & Comments \\
\hline A & Nominal mode & Four species $\left(\mathrm{H}^{+}, \mathrm{He}^{++}, \mathrm{O}^{+}\right.$, heavier ions), and energy-mass matrices periodically \\
B & Six species mode & Six species $\left(\mathrm{H}^{+}, \mathrm{He}^{++}, \mathrm{He}^{+}, \mathrm{O}^{+}, \mathrm{O}_{2}^{+}\right.$, heavier ions) \\
$\mathrm{C}^{*}$ & Light-ion resolution mode & Maximum mass resolution, optimized for low mass ion species $\left(\mathrm{H}^{+}\right.$to $\left.\mathrm{O}^{+}\right)$ \\
$\mathrm{D}^{*}$ & Heavy-ion resolution mode & Maximum mass resolution, optimized for high mass ion species $\left(\mathrm{O}^{+}\right.$to dust) \\
E & Energy band mode & Gives a subset of 16 steps from the energy scan. Maximum energy-mass resolution \\
F & Angular resolution mode & Optimized for high angular resolution, four species $\left(\mathrm{H}^{+}, \mathrm{He}^{++}, \mathrm{O}^{+}\right.$, heavier ions $)$ \\
\hline
\end{tabular}

*IMI can be operated in such a way that it alternates between modes C and D.

Table 3. Characteristics of the Ion Mass Imager.

\begin{tabular}{ll}
\hline Mass & $2.97 \mathrm{~kg}$ \\
Power & $8.0 \mathrm{~W}$ \\
$\begin{array}{l}\text { Energy } \\
\text { range (normal mode) }\end{array}$ & $10 \mathrm{eV} / \mathrm{q}$ to $35 \mathrm{keV} / \mathrm{q}$ \\
$\quad \begin{array}{l}\text { range (solar wind mode) } \\
\text { resolution }\end{array}$ & $100 \mathrm{eV} / \mathrm{q}$ to $10 \mathrm{keV} / \mathrm{q}$ \\
scan & $\Delta E / E=8 \%$ (one sector), 10\% (all sectors) \\
sector anomaly & $32 \mathrm{steps}(16 \mathrm{by}$ software integration) \\
Mass & $2.5 \%$ \\
range & 1 to $10^{12}$ a.m.u. in 32 mass channels \\
resolution & $\mathrm{see} \mathrm{Fig.} 4$ \\
scan & none (instantaneous) \\
Angular & \\
total field-of-view & $360^{\circ} \times 4.6^{\circ}$ \\
resolution azimuth & $20.0^{\circ} \times 4.6^{\circ}$ (in 16 azimuthal sectors) \\
Max. temporal resolution & \\
-2D & $0.5 \mathrm{~s}$ (time for one energy scan) \\
-3D & $4 \mathrm{~s} \mathrm{(half} \mathrm{a} \mathrm{spacecraft} \mathrm{spin} \mathrm{period)}$ \\
Geometric factor (per sector) & $3.5 \cdot 10^{-4} \mathrm{~cm}{ }^{2} \mathrm{sr}$ \\
\hline
\end{tabular}




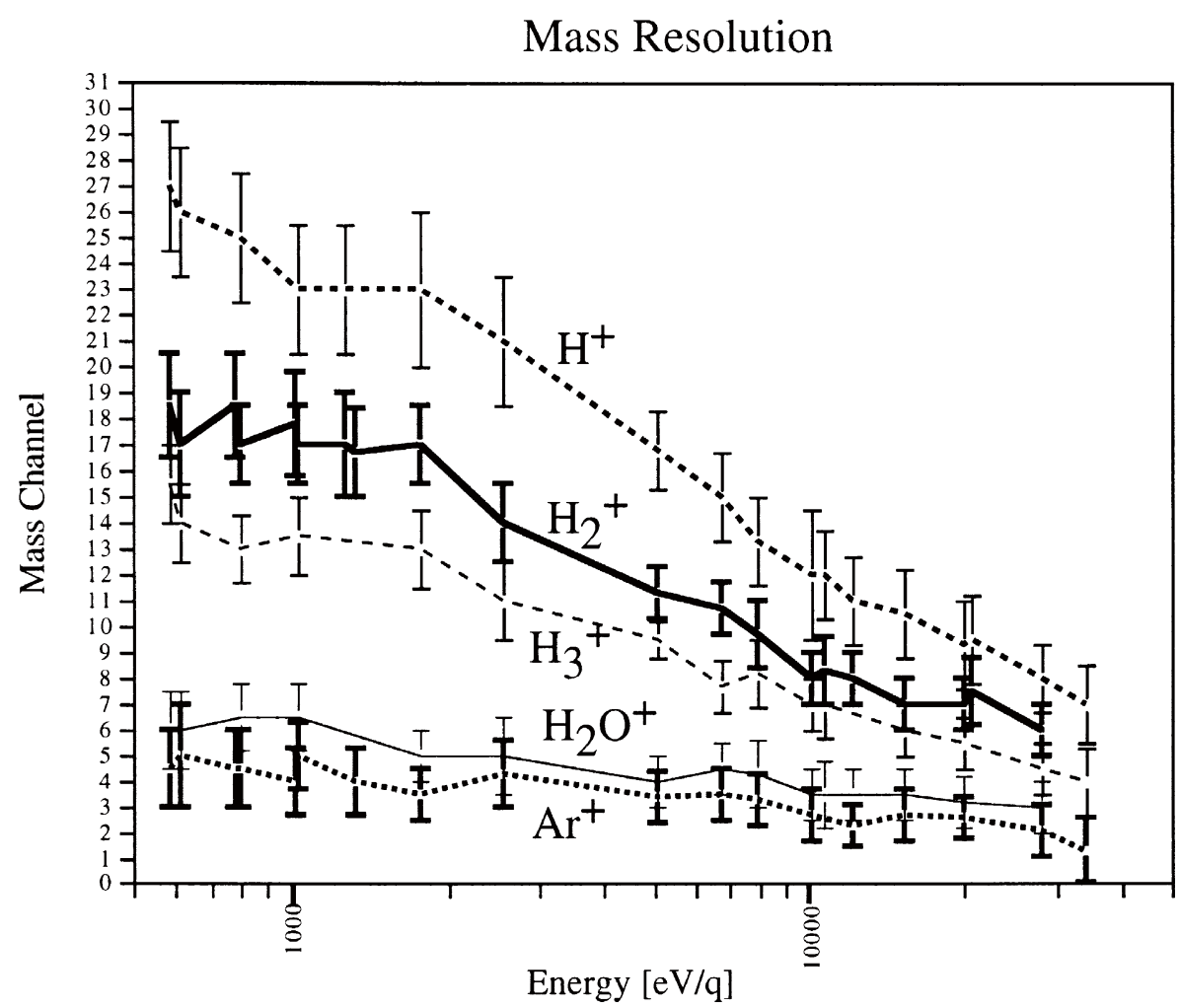

Fig. 4. Mass resolution of the IMI instrument as found during calibrations using the indicated ion species. The pre-acceleration voltage was set to 2.3 $\mathrm{kV}$, the setting used for all modes except mode D. The error bars for each mass gives the mass resolution.

of the opt-coupler also depends on the temperature. The temperature modulation is taken care by the software that set values of voltage. For example, we change the deflection settings by $-1 \mathrm{~V}$ every 7 degree the temperature increases to keep the real deflection voltage unchanged for different temperature.

\subsection{The electronics and data processing unit (IMI-E)}

The IMI electronics contains a Marconi MAS281 16-bit microprocessor for spectrometer control, commanding, telemetry interface, and on-board processing of the scientific data. IMI-E also contains four high-voltage power supplies used for the instrument. The instrument contains an EEPROM for software and constants, enabling updating of on-board software.

Since the mass imaging system produces $\approx 600 \mathrm{kbps}$ or one 16-bit mass-sector matrix ( 32 by 16 bins) every $13.6 \mathrm{~ms}$ sample interval, reduction and/or compression of the data must be performed before the data can be sent to the telemetry system. IMI uses a combination of data reduction, summation and loss-less compression. Initial (optional) data reduction transforms the 16-bit register values into 8bit values, according to either a logarithmic function or simply using the least significant 8 bits. Data summation can be performed over time, energy, mass channels, and angle (sectors). The data compression is based on the algorithm by Rice (CCSDS, 1994) and implemented in the on-board software.

A 2 Mbyte buffer allows for the instrument to produce more data than is being read out by the telemetry system during "active" periods with less compression efficiency. The data compression scheme is based on a target output data rate, and adjusts the pre-processing (data summation) only if the buffer becomes close to full. If the scheme has decreased the data output rate, it will readjust the preprocessing when the buffer is almost empty, to again reach the target data rate. The target data rates are between 6 bps and $12 \mathrm{kbps}$. For very low target data rates IMI performs computation of moments of the distribution function; calculating density, velocity vector, and pressure tensor for three selected ion species.

There are six operational mode groups (see Table 2) and some calibration modes. The instrument is commanded into a submode within a group, indicating a target data rate. The difference between the submodes in a group is the amount of pre-processing performed, mostly affecting the time resolution of the data. The instrument is then allowed to change the submode within the group in accordance to the data compression scheme. The energy-scans are synchronised with the spacecraft spin phase, and 16 scans are evenly distributed during a spin period. IMI will be operated in different modes depending on the spacecraft orbital phase.

\subsection{Calibrations and tests}

The final calibration of the flight instrument was performed in September-November 1997 in an ion source facility at IRF in Kiruna. This facility exposes the instrument to a wide (100 mm in diameter), homogenous beam of ions of various species (e.g., $\mathrm{H}^{+}, \mathrm{H}_{2}{ }^{+}, \mathrm{H}_{3}{ }^{+}, \mathrm{H}_{2} \mathrm{O}^{+}, \mathrm{Ar}^{+}$) within the energy range $20 \mathrm{eV} / \mathrm{q}$ to $50 \mathrm{keV} / \mathrm{q}$. A four-degrees-of-freedom turntable is used to determine the angular response of the instrument. See Table 3 for a summary of the IMI instrument characteristics, as measured during the calibration. Details of the calibration results are found in Yamauchi et al. (1998). 
Figure 4 shows the calibrated mass resolution of IMI using a $2.3 \mathrm{kV}$ pre-acceleration voltage. Sector anomaly is included in the figure.

\section{Summary}

IMI is a development of previous versions of similar instruments such as TICS on Freja which worked throughout the four year mission. The design principle is simple because of the use of permanent magnets for mass separation, and such simplicity provides the dependability necessary for interplanetary missions. The low mass/power requirements together with good instrument characteristics in terms of energy, mass, angular, and temporal resolution, also makes the instrument suitable for planetary space plasma physics missions. We have during the calibration of IMI found that the instrument has the capability to make a substantial contribution to the Planet-B mission, with detailed measurements of the ion composition in the Martian environment.

Acknowledgments. The IMI instrument was financed by grants from the Swedish National Space Board. The technical staff at the Swedish Institute of Space Physics are gratefully acknowledged for their dedicated work during the manufacturing and tests of the instrument.

\section{References}

Barabash, S. and R. Lundin, On a possible dust-plasma interaction at Mars, ISEE Transactions on Plasma Science, 22, 2, 173-178, 1994.

Barabash, S., E. Dubinin, N. Pissarenko, R. Lundin, and C. T. Russell, Picked-up protons near Mars: Phobos observations, Geophys. Res. Lett., 18, 1805-1808, 1991.

Barabash, S., R. Lundin, and O. Norberg, Upstream regions at Mars, in Plasma Environments of Non-Magnetized Planets, edited by T. I. Gombosi, pp. 285-290, Pergamon Press, Oxford, 1993.

Barabash, S., E. Kallio, R. Lundin, and H. Koskinen, Measurements of nonthermal helium escape from Mars, J. Geophys. Res., 100, 21,30721,316, 1995 .

CCSDS, Source Coding for Data Compression, Consultative Committee for Space Data Systems, CCSDS 111.0-W-2, Washington, D.C., March 1994.

Dolginov, Sh. Sh., Ye. G. Yeroshenko, and L. N. Zhuzgov, The magnetic field of Mars according to data from the Mars 3 and Mars 5, J. Geophys. Res., 81, 3353-3362, 1976.

Dubinin, E., R. Lundin, N. Pissarenko, S. Barabash, A. Zakharov, H Koskinen, K. Schwingenschuh, and Y. Yeroshenko, Indirect evidences for a gas/dust torus along the Phobos orbit, Geophys. Res. Lett., 17, 861864, 1990.

Dubinin, E., R. Lundin, H. Koskinen, and N. Pissarenko, Ion acceleration in the Martian tail: PHOBOS observations, J. Geophys. Res., 98, 39913997, 1993.
Dubinin, E. M., K. Sauer, R. Lundin, O. Norberg, K. Schwingenshuh, M. Delva, and W. Riedler, Plasma characteristics of the boundary layer of the Martian magnetosphere, J. Geophys. Res., 101, 27061-27075, 1996.

Eliasson, L., O. Norberg, R. Lundin, K. Lundin, S. Olsen, H. Borg, M. André, H. Koskinen, P. Riihelä, M. Boehm, and B. Whalen, The Freja hot plasma experiment-Instrument and first results, Space Sci. Rev., 70, 563-576, 1994.

Gringauz, K. I., V. V. Bezrukikh, M. I. Vergin, and A. P. Rezimnov, On the electron and ion components of plasma in the antisolar part of nearMartian space, J. Geophys. Res., 81, 3349-3352, 1976.

Intriligator, D. S. and E. J. Smith, Mars in the solar wind, J. Geophys. Res., 84, 8427-8435, 1979.

Ip, W.-H., On the oxygen corona of Mars, Icarus, 76, 135-145, 1988.

Ip, W.-H., T. K. Breus, and T. Zarnowiecki, Termination of the solar wind proton flow due to charge exchange effect near Mars, in Plasma Environments of Non-Magnetized Planets, edited by T. I. Gombosi, pp. 291-294, Pergamon Press, Oxford, 1993.

Lundin, R. and E. M. Dubinin, Phobos-2 results on the ionospheric plasma escape from Mars, Adv. Space. Res., 12, 9, 255-263, 1992.

Lundin, R., A. Zakharov, R. Pellinen, B. Hultqvist, H. Borg, E. M. Dubinin, S. Barabasj, N. Pissarenko, H. Koskinen, and I. Liede, First results of the ionospheric plasma escape from Mars, Nature, 341, 609612, 1989a.

Lundin, R., B. Hultqvist, S. Olsen, R. Pellinen, I. Liede, A. Zakharov, E. Dubinin, and N. Pissarenko, The ASPERA experiment of the Soviet phobos spacecraft, in Solar System Physics, edited by J. H. Waite, Jr., J. Burch, and T. E. More, pp. 417-424, AGU, Washington, D.C., $1989 b$.

Lundin, R., A. Zakharov, R. Pellinen, B. Hultqvist, H. Borg, E. M. Dubinin, S. Barabasj, N. Pissarenko, H. Koskinen, and I. Liede, Plasma composition measurements of the Martian magnetosphere morphology, Geophys. Res. Lett., 17, 877-880, 1990.

Lundin, R., E. Dubinin, S. Barabash, and O. Norberg, ASPERA observations of Martian magnetospheric boundaries, in Plasma Environments of Non-Magnetized Planets, edited by T. I. Gombosi, pp. 311-320, Pergamon Press, Oxford, 1993.

Riedler, W., et al., Magnetic fields near Mars: First results, Nature, 341, 604-607, 1989.

Vaisberg, O. L., Mars-Plasma environment, in Physics of Solar Planetary Environment, Vol. 2, edited by D. J. Williams, pp. 854-871, AGU, Washington, D.C., 1976.

Vaisberg, O. and V. Smirnov, The Martian magnetotail, Adv. Space Res., 6, 301-314, 1986.

Yamauchi, M., S. Olsen, H. Borg, O. Norberg, M. Hirahara, R. Lundin, and K. Lundin, Report on the Calibration of the IMI Instrument for Planet-B, IRF Technical Report, Swedish Institute of Space Physics, Kiruna, Sweden, 1998 (in press).

Yeroshenko, Ye., W. Riedler, K. Schwingenschuh, J. G. Luhmann, M. Ong, and C. T. Russell, The magnetotail of Mars: PHOBOS observations, Geophys. Res. Lett., 17, 885-888, 1990.

O. Norberg (e-mail: olle.norberg@irf.se), M. Yamauchi, R. Lundin, S. Olsen, H. Borg, S. Barabash, M. Hirahara, T. Mukai, and $\mathrm{H}$. Hayakawa 